\title{
A Dermatologia na Enfermaria de Medicina Interna: Análise Retrospectiva de um Centro Terciário
}

\author{
Pessoa e Costa, Tomás'; João, Ana Luísa'; Pereira, Márcia²; Estriga, Ana Rita²; Rocha Páris, Filipa'; Gomes da Silva, Eduardo² \\ 'Serviço de Dermatologia do Hospital Santo António dos Capuchos, Centro Hospitalar Lisboa Central, Lisboa, Portugal \\ 2Serviço de Medicina Interna 2.3 do Hospital Santo António dos Capuchos, Centro Hospitalar Lisboa Central, Lisboa, Portugal
}

RESUMO - Introdução: As doenças da pele são comuns nos doentes internados nas enfermarias de Medicina Interna e requerem frequentemente a observação por um dermatologista. Este estudo pretende caracterizar o padrão de patologia cutânea destes doentes, avaliando o impacto da Dermatologia na sua abordagem. Métodos: Foi realizado um estudo retrospectivo dos pedidos de observação por Dermatologia nos doentes internados nos Serviços de Medicina Interna do Hospital Santo António dos Capuchos, ao longo de 46 meses. Os diagnósticos associados a esses pedidos foram classificados de acordo com a $10^{a}$ revisão da Classificação Estatística Internacional de Doenças e Problemas Relacionados à Saúde. Resultados: Foram observados 231 homens e 217 mulheres, com uma idade média de 72,6 anos. As dermites de estase e as úlceras de perna venosa foram o diagnóstico mais comum. Discussão e Conclusão: A equipa de Dermatovenereologia diminuiu significativamente o número de doentes sem diagnóstico. O nosso estudo parece reforçar a importância dos dermatologistas em contexto hospitalar.

PALAVRAS-CHAVE - Dermatologia; Hospitalização; Medicina Interna.

\section{Dermatology in Internal Medicine Wards: Retrospective Analysis of a Tertiary Center}

\begin{abstract}
Background: Skin diseases are common in Internal Medicine wards and often require observation by a dermatologist. This study intends to characterize the pattern of skin pathology in these patients and to evaluate the impact of dermatologists in their approach. Methods: A retrospective study was conducted. Dermatology observations over 46 months in Internal Medicine wards of Hospital Santo António dos Capuchos were analyzed. Patients' conditions were classified according to the 10th revision of the International Statistical Classification of Diseases and Related Health Problems. Results: 231 men and 217 women were observed, with an average age of 72.6 years. Stasis dermatitis and venous leg ulcers were the most common diagnosis. Discussion and Conclusion: The Dermatology team significantly reduced the number of undiagnosed patients. Our study reinforces the importance of dermatologists in the evaluation of inpatients with skin diseases.
\end{abstract}

KEYWORDS - Dermatology; Hospitalization; Internal Medicine.

\section{INTRODUÇÃO}

A evolução e melhoria no tratamento das principais doenças da pele, com consequente redução do número de doentes internados nos serviços de Dermatovenereologia, tem levado alguns autores a questionar o papel desta especialidade em contexto hospitalar. ${ }^{1}$ No entanto, outros têm defendido que a importância da Dermatologia Hospitalar não se esgota no seu número de internamentos, realçando que os dermatologistas são frequentemente requisitados para a observação de doentes internados noutras especialidades, que no decurso da sua hospitalização desenvolvem patologia cutânea ou exacerbações das suas doenças da pele. ${ }^{2-13}$

Desta forma, este estudo pretende caracterizar as observações prestadas ao longo de 46 meses por um serviço de Dermatovenereologia de um hospital terciário, a doentes internados nas enfermarias de Medicina Interna desse mesmo hospital. É também nosso objectivo identificar as principais características epidemiológicas desta população e o seu padrão de doenças da pele. Pretendemos ainda
Correspondência: Tomás Pessoa e Costa Serviço de Dermatologia

Hospital de Santo António dos Capuchos - CHLC

Alameda de Santo António dos Capuchos

1169-050 Lisboa, Portugal

E-mail: tomaspessoaecosta@gmail.com

DOl: https://dx.doi.org/10.29021/spdv.78.1.1099 (c) Autor (es) (ou seu (s) empregador (es)) e Revista SPDV 2020. Reutilização permitida de acordo com CC BY-NC. Nenhuma reutilização comercial.

(c) Author(s) (or their employer(s)) and SPDV Journal 2020. Re-use permitted under CC BY-NC. No commercial re-use. 


\section{Artigo Original}

avaliar o impacto da observação pela equipa de Dermatologia, comparando os diagnósticos inicialmente propostos pela equipa de Medicina Internas e os diagnósticos finais propostos pelo dermatologista.

\section{MÉTODOS}

Foram recolhidos de forma retrospectiva, com base nos registos informáticos, todos os pedidos de colaboração submetidos pelos diversos serviços de Medicina Interna que compõem o Hospital Santo António dos Capuchos (Lisboa, Portugal), ao serviço de Dermatovenereologia desse mesmo hospital, efectuados entre Julho de 2014 e Maio de 2018 (46 meses). Foram excluídos da análise os casos associados a um registo informático incompleto.

Para caracterização epidemiológica, foi registado o sexo e idade de cada doente. Registou-se ainda o tempo compreendido entre o pedido de colaboração e a observação presencial do doente; o diagnóstico inicialmente proposto pelos médicos do serviço de Medicina Interna; e o diagnóstico proposto após observação da Dermatovenereologia. Os diagnósticos foram depois codificados com base na $10^{a}$ revisão da Classificação Estatística Internacional de Doenças e Problemas Relacionados à Saúde (ICD-10) e foram comparados os resultados entre as duas especialidades.

\section{RESULTADOS}

Ao longo de 46 meses, foram solicitados 551 pedidos de observação ao serviço de Dermatologia. Vinte casos foram excluídos da análise pois o registo informático estava incompleto. Três pedidos foram excluídos por falecimento do doente no dia em que foi pedida a consulta.

Foram observados 448 doentes - 231 homens e 217 muIheres, num total de 528 observações. A idade média dos doentes foi de 72,6 anos (intervalo 19-100; desvio padrão $15,31)$. Só 3 observações não foram prestadas no próprio dia do pedido $(0,06 \%)$. Uma única observação pela equipa de Dermatologia foi considerada suficiente em $72,7 \%$ dos doentes (média de observações por doente de 1,18).

Em 289 dos pedidos de observação analisados (54,7\%), a equipa de Medicina Interna não tinha formulado hipóteses de diagnóstico. Em 3 casos já não foram documentadas lesões aquando da observação pela Dermatologia. Dos restantes doentes, apenas 6,5\% $(n=34)$ ficou sem diagnóstico após observação por um dermatologista. Para além disso, a equipa de Dermatologia também alterou o diagnóstico inicialmente proposto pela Medicina interna em 25 casos.

Segundo a classificação ICD-10, as úlceras venosas de perna (I83.2) foram o diagnóstico mais frequente nos doentes observados pela Dermatovenereologia, afectando $12,7 \%$ dos doentes $(n=57)$ e em estreita relação com as dermites de estase (183.1), que afectaram 36 doentes (8\%). Outros diagnósticos frequentes incluíram as toxidermias (L27.1) com 29 casos (6,5\%); as celulites do membro inferior (L03.1) com 27 casos (6\%); e a psoríase (L40.0) com 26 casos $(5.8 \%)$ (Tabela 1$)$.

\section{DISCUSSÃO}

A caracterização das doenças da pele nas enfermarias de Medicina Interna tem sido estudada por alguns autores. $^{2,4,5,11,13}$ De facto, tem sido defendido que as dermatoses inflamatórias e infecciosas são mais frequentes nos doentes internados, ${ }^{2,4,5,11}$ pelas alterações dos hábitos de cuidados da pele e de higiene inerentes ao próprio internamento, ${ }^{2,4}$ bem como pelo elevado número de doentes imunodeprimidos nestas enfermarias. ${ }^{2}$ Os nossos resultados corroboram estes achados e colocam em evidência, também, o elevado número de pedidos de observação motivados por úlceras de perna de etiologia venosa. O agravamento destas úlceras em contexto de internamento está em provável relação com a alteração dos cuidados diários de penso e reforça a necessidade de uma colaboração ampla entre especialidades, que envolva também a equipa de enfermagem.

Em 2012, Fernandes et $a l^{12}$ analisaram as doenças da pele dos doentes internados no Hospital de Santo António, Centro Hospitalar do Porto (Porto, Portugal), ao longo de um ano. Aumentando o número de doentes observados e o tempo do estudo (e apesar da nossa análise só envolver doentes de Medicina Interna), os nossos resultados corroboram, na sua generalidade, o único estudo publicado para a população portuguesa.

A relação da Medicina Interna e da Dermatologia tem sido frequentemente debatida. De facto, em 1998 Lynch $^{1}$ admitia que os doentes com doenças da pele grave deviam ser internados ao cuidado de internistas, pela sua maior experiência na gestão das suas múltiplas complicações. No entanto, muitos autores têm refutado esta ideia, defendendo que a abordagem destes doentes por médicos não-dermatologistas

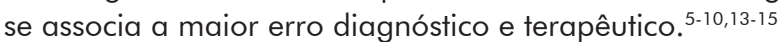

Os nossos resultados mostram que a observação por Dermatologia reduziu significativamente o número de doentes sem diagnóstico. Mostram ainda que, na maioria dos casos, esta melhoria na acuidade diagnóstica foi conseguida através de uma única observação. No entanto, estes achados devem ter em conta algumas limitações do nosso estudo, nomeadamente a sua natureza retrospectiva, o recurso à avaliação do dermatologista como método de "gold standard" de diagnóstico e a difícil comparação com outros estudos, pela pouca utilização de escalas de classificação de doença internacionalmente validadas.

Apesar destas limitações, o nosso estudo reforça a importância do dermatologista na abordagem dos doentes com doenças da pele, mesmo que internados noutros serviços.

\section{CONCLUSÃO}

A observação por um dermatologista é muitas vezes solicitada em doentes internados nas enfermarias de Medicina Interna. As dermatoses inflamatórias e infecciosas são frequentes nestes doentes. Na maioria dos casos, uma única observação pela equipa de Dermatologia associou-se a uma melhoria significativa da acuidade diagnóstica, o que reforça a importância desta especialidade em contexto hospitalar. 


\section{Artigo Original}

Tabela 1 - Diagnósticos mais frequentes na população observada.

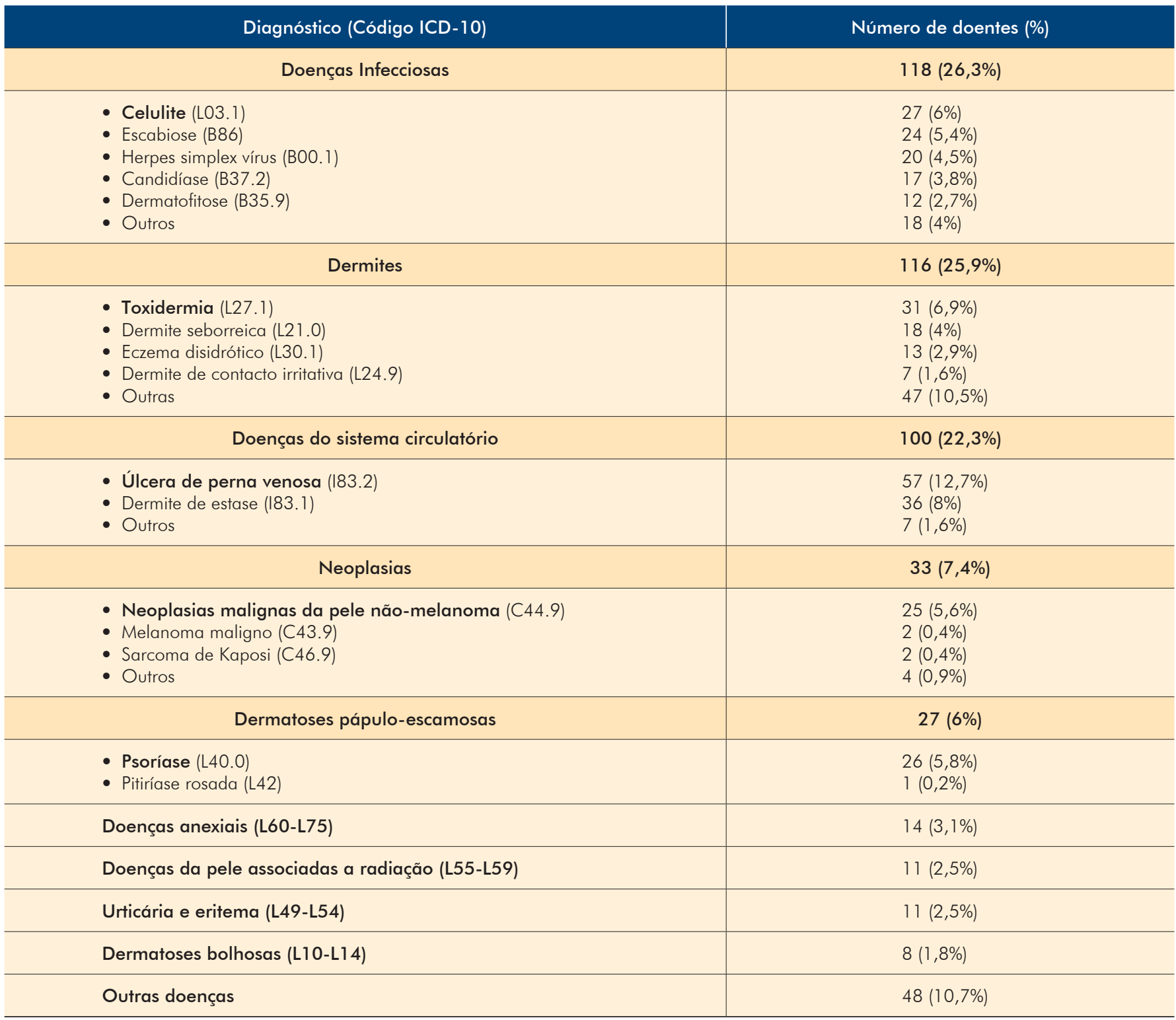

Conflitos de interesse: Os autores declaram a inexistência de conflitos de interesse na realização do presente trabalho.

Fontes de financiamento: Não existiram fontes externas de financiamento para a realização deste artigo.

Confidencialidade dos dados: Os autores declaram ter seguido os protocolos da sua instituição acerca da publicação dos dados de doentes.

Protecção de pessoas e animais: Os autores declaram que os procedimentos seguidos estavam de acordo com os regulamentos estabelecidos pelos responsáveis da Comissão de lnvestigação Clínica e Ética e de acordo com a Declaração de Helsínquia da Associação Médica Mundial.

Proveniência e revisão por pares: Não comissionado; revisão externa por pares.
Conflicts of interest: The authors have no conflicts of interest to declare.

Financing support: This work has not received any contribution, grant or scholarship.

Confidentiality of data: The authors declare that they have followed the protocols of their work center on the publication of data from patients.

Protection of human and animal subjects: The authors declare that the procedures followed were in accordance with the regulations of the relevant clinical research ethics committee and with those of the Code of Ethics of the World Medical Association (Declaration of Helsinki).

Provenance and peer review: Not commissioned; externally peer reviewed 


\section{Artigo Original}

\section{REFERÊNCIAS}

1. Lynch PJ. The Future of Medical Dermatology. Arch Dermatol. 1998; 134:358.

2. Mancusi S, Festa Neto C. Inpatient dermatological consultations in a university hospital. Clinics. 2010;65:8515.

3. Alani A, Sadlier M, Uddin A, Hackett C, Ramsay B, Ahmad K. An analysis of inpatient dermatologic consultations at University Hospital Limerick: inadequate infrastructure leads to acute skin failure. Ir J Med Sci. 2017;186:305-7. doi: 10.1007/s1 1845-016-1424-8.

4. Peñate $Y$, Guillermo N, Melwani P, Martel R, Borrego L. Dermatologists in hospital wards: an 8-year study of dermatology consultations. Dermatology. 2009;219:22531.

5. Tay LK, Lee HY, Thirumoorthy T, Pang SM. Dermatology referrals in an East Asian tertiary hospital: a need for inpatient medical dermatology. Clin Exp Dermatol. 2010;36:129-34.

6. Koh H. A retrospective analysis of dermatological problems in a hematology ward. Clin Cosmet Investig Dermatol. 2013;6:145-9. doi: 10.2147/CCID.44853.

7. Kirsner RS, Yang DG, Kerdel FA. Inpatient dermatology. The difficulties, the reality, and the future. Dermatol Clin. 2000; 18:383-90, vii.

8. Falanga V, Schachner LA, Rae V, Ceballos PI, Gonzalez $A$, Liang $G$, et al. Dermatologic consultations in the hospital setting. Arch Dermatol. 1994;130:1022-5.

9. Nahass GT. Inpatient dermatology consultation. Dermatol Clin. 2000;18:533-542, x.

10. Raikar DR, Manthale NS, Raikar SR. Inpatient dermatological referrals in a tertiary care hospital. Int J Res Dermatol. 2017;3:251.

11. Feldman SR, Fleischer AB Jr, McConnell RC. Most common dermatologic problems identified by internists 1990-1994. Arch Intern Med. 1998;158:726-30.

12. Fernandes IC, Velho G, Selores M. Dermatology inpatient consultation in a Portuguese university hospital. Dermatol Online J. 2012;18:16.

13. Galimberti F, Guren L, Fernandez AP, Sood A. Dermatology consultations significantly contribute quality to care of hospitalized patients: a prospective study of dermatology inpatient consults at a tertiary care center. Int J Dermatol. 2016;55:e547-51. doi: 10.1111/iid.13327.

14. Hu L, Haynes H, Ferrazza D, Kupper T, Qureshi A. Impact of specialist consultations on inpatient admissions for dermatology-specific and related DRGs. J Gen Intern Med. 2013;28:1477-82. doi: 10.1007/s11606-0132440-2.

15. Kroshinsky D, Cotliar J, Hughey LC, Shinkai K, Fox LP. Association of dermatology consultation with accuracy of cutaneous disorder diagnoses in hospitalized patients: a multicenter analysis. JAMA Dermatol. 2016;152:47780. doi: 10.1001/jamadermatol.2015.5098. 